\title{
A Permanent Cure for Vitiligo: Immigration of Healthy Skin Cells to Unhealthy Sides of the Skin in Vitiligo through a New Treatment
}

\author{
Akbar Mohammadrezaei ${ }^{1 *}$, Nooshin Mohammadiasl ${ }^{2}$ \\ ${ }^{2}$ Doctor and Researcher \\ n.masl12@yahoo.com \\ *Corresponding Author: Akbar Mohammadrezaei, Doctor and Researcher, Urmia, Iran.
}

Abstract

A Permanent Cure for Vitiligo: Immigration of Healthy Skin Cells to Unhealthy Sides of the Skin in Vitiligo

This article is about a skin disorder which manifests itself as white patches (pigment destruction) in different locations of the body. There are many classifications for this disorder such as segmented, focal, generalized and universal vitiligo. In my article, I have mentioned some of the current methods used in the treatment such as UV rooms. However, there are many risks and long term side effects to this treatment. Besides at the end of the treatment, the patient leaves the clinic in disappointment and without being treated. I was suffering from the same disorder for 14 years, so I can feel how deep is the depression and suffering of those vitiligo patients. My motivation behind the discovery of this treatment was me, myself. I was suffering from the same disorder. It was so painful to be out in the society where everybody gazed at me and some were even afraid to talk to me. Because of my economic issues, I did not have access to labs and research centesrs. Consequently, my only opportunity was to do self-study and broadened my understanding and knowledge about human body and biology. Thanks to those studies, I cured myself in 5months and then many more patients from England, Iran, Turkey and Azerbaijan. In my article, I have written about some blood tests the patients have to take. I have also mentioned the vitamins, pills and the creams they should use(different for kids and adults). My only intention for writing this article is to let all my dermatologist colleagues know about the new treatment. Moreover, the whole treatment takes 5 months or so and is affordable by everybody. If the patients follow the simple advice mentioned in the article, the disorder will not come back. I would kindly ask you to go through my article and read it deep. I have treated myself and many others, so I can replicate the treatment on the cases given by you. The treatment and progress is visible from the first month.

\section{INTRODUCTION}

\section{Vitiligo:Treatment with a New Method and Quick Recovery}

Vitiligo is a disease in which the pigment cells of the skin are destroyed in some areas. The symptoms include loss of skin color, manifesting itself as white patches and macules in different locations in the body. However, repigmentation or its sudden and spontaneous recovery is very rare. In the half of the cases reported, the disease begins to advance in the skin before the age of 20 . Unfortunately, these people face psycological pressure in important stages of their lives, such as job opprtunities, marriage and studying, specially if it is conjugated with some other disease such as hypothyroidism, diabetes, mellitus and rheumatisim. Vitiligo may exacerbate with factors such as malnutrition, sunburn, skin trauma, stress, psychological tension, pregnancy and long term contact with chemical combinations used in painting.

There are three ways to prevent melanin production:

1. To stop the activity of thyrosinase enzyme

2. To prevent the process of transferring of melanosoms to keratinocytes

3. To destroy melanis or melanocytes 


\section{MATERIALS AND MeThodS}

In different resources, based on clinical evaluations, there are various distinctions for this disease. As written in Autoimmne Melanocyte Destruction in Vitiligo, "clinical presentations include, (a) segmental vitiligo; characterized by lesions that occure in a dermatomal, symmetric distribution ( of limited clinical signifcance); (b) focal vitiligo, characterized by a limited number of small lesions; (c) generalized vitiligo, the most common type of vitiligo, where lesions occur with bilateral, symmetrical distribution and (d) universal vitiligo, indicating complete or almost complete depigmentation" $(81,1061-1068$, 2001).

Many theories regarding the causes of this disease have been introduced. One of them in Function Blocking Autoantibodies to the Melanin is as follows,

1. The theory of neuro chemical, ie, the release of toxin from the end or tail of the nerves, decrease in melanin production and consequently destruction of melanocyts.

2. The theory of biochemical, ie, increase in the synthesis inductor productions of melanin toxic, decrease in the number of defensive free radicals, mounting production of proxid hydrogen, and consequenty spontaneous destruction of the pigments.

3. Autoimmune disease due to the production of antibodies against melanocytes( $86,781-784$, 2006).

Other theories involve the amount of iron and copper in the body. One of these theories has been mentioned in an Iranian Medical Magazine as , "there is a close connection between the activity of the cells and the amount of the copper and zinc ions, and in a lower degree the amount of cobalt, nickel and vanadium in nerve cells. Copper ions have a role as co-factor of dopamine betahydroxilase enzyme and are important in regulationg the metabolism of monoamins. They also have a minor role in the function of thyrosinase enzyme. Accumulation of neurotoxic amyloid neuropeptides causes an increase in the ionic level of calsium inside the neuron and this consequently damages the functions of neurons. The increase in the level of zinc ion can act as a factor to accelerate the accumulation of peptide beta amyloid and the disorder in calcium dependent potasium chanels can lead to an increase in the amount of zinc ion" $(43,2003)$.

Current introduced and applied treatments, such as using oxsoralen combinations, UV rooms, local cellular culture and injection lead to weakess in immune system and haven't been able to produce acceptable clinical results. Unfortunately,I didn't have access to laboratory and research centers, so based on my own clinical observations and self studies, I discovered more about the patobiology of this disorder. Relying on those studyings and my findings, I cured many people suffering from vitiligo. However, at the begining days of treatment procedure, I didn't record my patients' names I also didn't take their before-after pictures to make an organized list. However, I have attached some of the pictures I took later on. As time passed, and I saw that the treatment is working and people are cured, I decided to introduce this treatment to the world. Since 15 April, 2018 I have started to register my patients' names, age, involved areas of the skin, the time span of affiction, their phone numbers and photos.

I don't completely agree with biochemical theory and this is because of the many stressful processes the patient undergoes, such as psychological pressure, traumatic stress, stress caused by malnutrition, contact with chemical combinations used in farming and painting. Furthermore, the balance of microelements such as copper and zinc- in the locations where malanins and neurons are connected- is distracted and the amount of micro circulation and oxygen accessible to the cells reduces. Consequently, the body produces and releases metabolites unknown to the immune system. The body' s weak immune system in confronting free radicals, begins to damage toxic element producing cells, and this leads to gradual decrease, stop and destruction of melanocytes in that area. The triggering elementof these chain like reactions is the declaring factor of the type, intesnsity and time span needed for the treatment.

Now, if we try to balance all micro elements in the whole body- with more emphasis on the micro elements of the involved areas- and to increase the amount of oxygen crucial and needed for the cells and to motivate the healthy neighbouring cells to immigrate (move) to kratinocytes of the involved and afflicted areas, we will witness the recovery. However, 
in patients above $55 \%$ affliction and also those who come to start the tratment after along time of their affliction, the best way will be to create micro element balance, to better micro circulation and to eliminate the healthy melanocytes in order to achieve an even skin tone without any color contrast.

\section{Treatment in Practice}

Firstly, I ask them to do these examinations:

1) CBC with diff- FBS and HbA1C- AST and ALT

2) BUN and Crea- TSH and FT4 and Free- T3 and Ferritine

Secondly, having checked the results of these two examinations, if there is a concurrent disorder, I treat that disorder before I start the treatment related to vitiligo. What I perscribe to my patients are:

1. Capsul para amino benzoic acid. This capsul should be taken daily- until the last day of the recovery. According to Daroyab Website,"Paba with the formula, $\mathrm{H} 2 \mathrm{NC} 6 \mathrm{H} 2 \mathrm{CO} 2 \mathrm{H}$ is an important inductor product of building folat and is easily absorbed by the small intestine. PABA, causes the consumption of cellular oxygen and also, through interference of mono amino oxidase, increases the decomposition of serotinin or hydroxytriptamine.Serotininis a kind of neurotic transmitter from the group of amino acid biologic and one of the derivatives of tripotofan" (G4538). It can be found in liver, brewers yeast, kidney, molasses, mushrooms, whole grains and unfiltered beer.

2. Multi- vitamin capsules for 1 month. From the second month, I add body cleansing capsules, the patient takes it for 1 month. After the third month, until the last day of the recovery, I perscribe spirulina capsul one in a day.

3. Furthermore, I perscribe chlordiazepoxide pill, two times a day. This totally depends on the living conditions of the patient. If they are living in circumstances surrounded by stress, I perscrib it to them. However, depending on the patients decision, I might also not perscribe it.

4. Hypiran edible herbal drop. The patient- every 8 hour, takes 12 drops of it. This will continue to the last day of the recovery. This herbal drop is made from the extract of a plant called Hypericum perforatum and includes $25 \%$ hypercin. Through regulating mono amino oxid enzyme, it has a role as an anti stress and soothening element.

5. The most important part of the treatment process is the pharmaceutical combination of a pill from the family of psoralenes and a cream from the family of corticostroides. This mixture and its percentage depend on the intensity of the afflicted areas on skin. Based on my own findings and studies, I believe, contrary to the current treatment methods, the lotion or solution mode of the pill must not be used. Moreover, the afflicted areas being treated must not be exposed to sun light or UV lamps and absolutely must be protected by sunscreens above $50 \mathrm{SPF}$. The mixture should be externally applied on afflicted areas on skin before bedtime. The next day, in the morning, immediately after waking up, the cream must be washed off with shea butter soap.

Corticostroids steroidswith entering the core of the cell can change the process of RNA transcription. They can also change, motivate or stop the synthesis of some particular proteins. Moreover, by preventing the accumulation of inflammated cells, they stop phagocytosis.They also cause the realese of enzymes responsible for inflammation. The pill derivated from pesoralen, stabilizes epiderm cells and increases the activity of thyrosinase in the cells which produce melanin (In order to produce melanin from the oxidation of thyrosine, we need the enzyme thyrozinase).

6. Shea butter soap. For morning wash of the areas involved. The patients apply it to the afflicted areas, wait for 2 minutes and then rinse it off with water.

7. They mix powder of two herbs, a mineral edible substance $(500 \mathrm{mg}$ for adults and $250 \mathrm{mg}$ for children between 3 and 12) and honeyin a glass of warm water and drink it half an hour before breakfast.

Shea butter is extracted from the seed of shea butter and its original name is Butyraspermum Parkii. It is native to East Africaand lives for 200 years. Shea butter extract includes unsaturated, non soap oils, essential fatty acids, phytostroles, vitamin $\mathrm{E}$ and $\mathrm{D}$, provitamins and alantoine. Its effects on skin are as following: 
a) It stimulates external micro circulation of the skin.

b) Itincreases skin resistanceagainstenvironmental pollution.

c) It protects the skin from UV rays.

d) It renovates the skin and stimulates clogen production.

e) It has anti inflammation effects.

f) With its anti oxydant features, it prevents and reduces wrinkles in the skin.

8. Emphasized use of sunscreen, specifically on the areas affected by vitiligo and the areas on which pharmaceutical combination is applied(from the first day of treatment untill the last day of recovery, a sunscreen with SPF above 50).

9. Vitamin C cream, with the form of lyposomal.
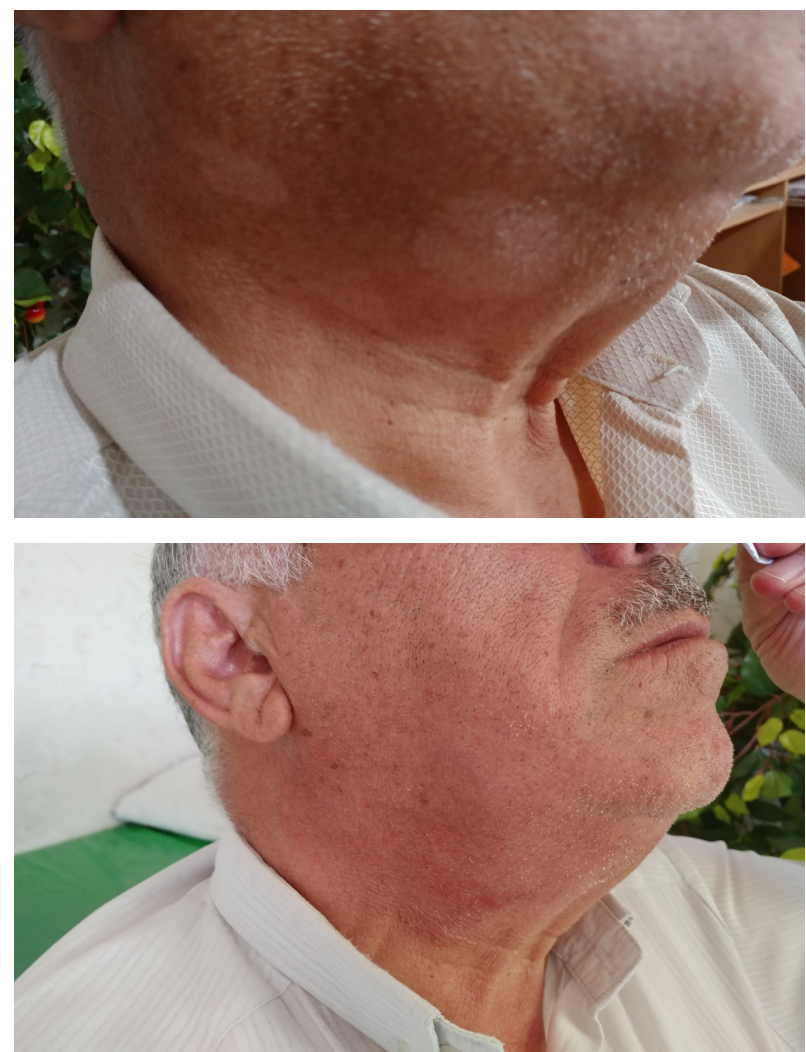

1 month and a half after the first visit, I ask my patients to come again so that I can check the amount and the speed of the recovery. At this time, the patients can observe the movement of the cells of the healthy areas toward the afflicted parts of the skin. This is visible as brown spots in hypopigmented areas. If I can't see the track of recovery at this time, I change the intensity of the pharmaceutical combination I perscribe. However, in the majority of my patients there is no need for this change. 3 months after the second visit, the recovery is very obvious and visible to the patients.
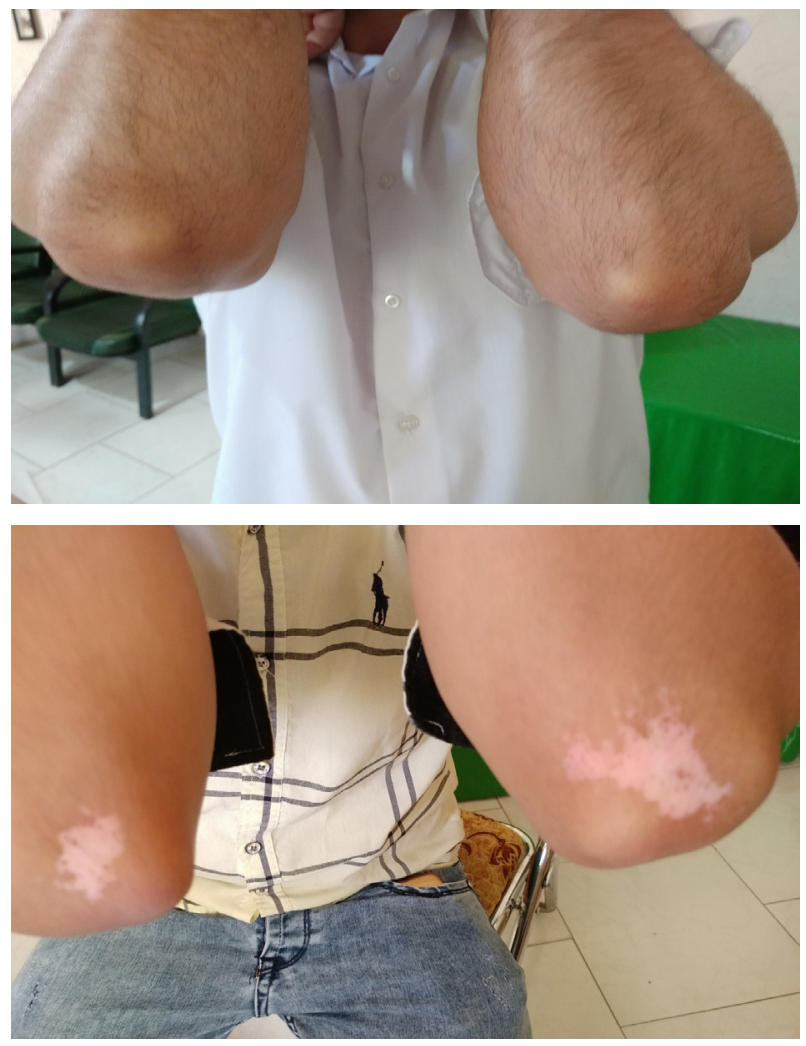

Considering the generalized type of vitiligo( above $55 \%$ ), I prescribe body cleansing capsul one daily for a period of 1 month, along with spirulina capsul one daily for a period of 3 months. After the second month, I add supplus meds capsuls and zinc plus, one from each daily for a period of 1 month. Concurrently, I perscribe the pharmaceutical combination for external use, 2times a day, in the mornings and evenings. They apply this cream on healthy looking areas of the skin( the reason is that these healthy looking parts of the skin have been exposed to unhealthy skin cells. The result of this damaging exposure might manifest itself years later). Regular daily shower with shea butter soap is another important factor affecting the process of treatment and recovery. Through pulstherapy, it accelerates the process of recovery. Some patients complain about slight itchiness orwrinkled areas of skin. I just ask them to be more patient as it won't do any serious harm and won't affect the process of recovery negatively. 
I should emphasize that during the time the patients are wearing the combination cream, they shouldn't wear sunscreen( If the sunlight is not very strong). The reason is that if they wear it on their skin, exposure to light and weak sunlight will stimulate the motivation of melanocytes and this consequently leads to better absorbment of the cream and progress in treatment.
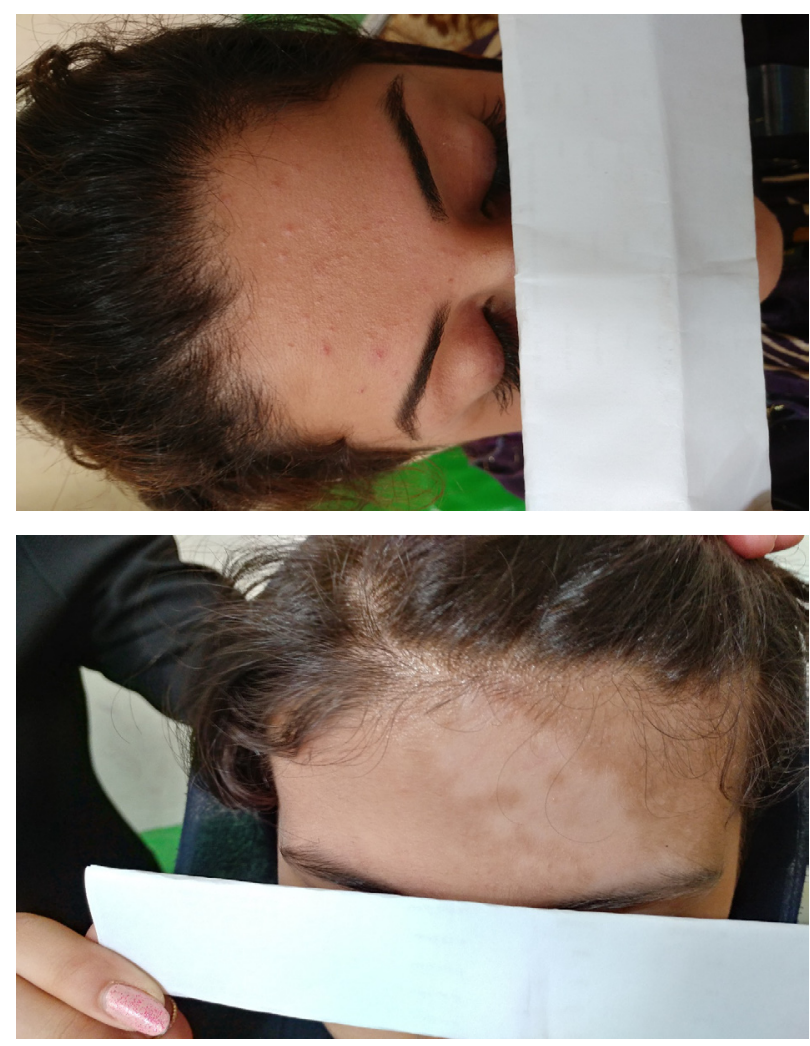

The whole treatment period takes a time between 4 and 5.5 months. Even after the recovery, the patients should wearsunscreen. They willalso continuewearing combined cream for 3 months after being recovered, once in a week. The reason is that some melanocytes might be in silent mode ( off mode) and might suddenly become active after a short while after the recovery period and cause somehyperpigmentationareas. The patients do not have to continue taking any pills or any other creams. Some of the patients ask about the change in the hair color of the areas where they apply combined cream. I can firmly inform them that the hair color won't change. They also ask questions about the recovery time period. Considering focal type, it might take more than a year and a half and the generalized type will take less than 5 months and a half. They also pose questions like: Will vitiligo come back? I tell them If you follow these, it will never return.
1) Sunlight through the eyes or skin will have a negative effect. Patients should wear sunscreen, and sunglassesUV 400 or photo chromic $60 \%$. Usually, vitiligo advances faster in summer and spring. This is because the color contrast in the margin of the vitiligo spots increases a lot at this time of the year. Also the sunlight is absorbed and eyes retina delivers stimulated electric puls through vision neurons. This increases the activity of Pineal in brain, melatonin production, abnormal and toxic inductors in body.

2) Stay away from stressful environments.

3) Have early night sleep and follow cirocadian rhythm.

4) Use green vegetables in your diet more, such as geonia, cori andrum stavium, parsely, petroselinum crispum, vigna mungo.

5) No alcholic drinks.

\section{ConClusion}

Contrary to the current methods, the technique I use is safe and permanent. The current introduced theories have long lasting physical and psychological side effects. In my treatment, I try to create a balance in microelements neighbouring the melanocytes. I also try to improve microcirculation and increase the cellular oxynazation. All of the patients who have referred to me have totally recovered, including 2 citizens from England, some more from Turkey, Azerbaijan and many more from Iran. I hope, with the help of dermatology centers and organizations, I can develope this technique and the medicine to be produced as one pill and one cream. As for now, I use some vitamines, creams and pills( I take different ingredients needed for the treatment from different vitamines, drugs and creams). With your help and support, I can take the needed ingredients, put them all in one or two pills and combine all the creams to make just one, easily applicable and affordable to everyone suffering from it.

All my patients, desperately, asked me to introduce the treatment to the world, to all those suffering from it, as they have tasted the pain of this disorder and the suffering to the last bit. They were the real motivation behind this article and my interest in informing other dermatologists about this treatment. I have the before and after pictures of my patients from April 15, 2018. Pictures belonging to dates before the mentioned day 
are not available, as I was not thinking of publishing my treatment officially. If you dear collegues are interested in my treatment, I can even replicate it on the cases offered from you in your company and under your observation. 2 months will be enough for the recovery to be visible.

I believe patients out there need us desperately, they need your support of this treatment. Their prays are with us, and maybe this treatment is an answer to their prays. They are hopeful, they hope for it every second. We should hear them. Now we have found the cure and it is our humanitarian responsibility to let everyone know about that. We should assist them to beat the disorder and say hello to a happier life.

\section{ACKNOWLEDGMENT}

For years and years, I suffered from vitiligo disorder. I was feeling embarrassed when I walked in the street. I had a more terrible feeling when I went to my children's school to pick them or when I attended the parents meeting. My children Ali and Maryam and more than them, my true love and wife, Fatemeh treated me so nicely. They were with me during those bitter years. I would love to thank them all for their patience and long lasting love toward me. They were by my side during those years and during the treatment. They blew hope to my heart and backed me in all those desperate moments of my life. I am fine now but I want to let them know that I will not forget their sacrifice, love, patience and devotion. I would love to thank Nooshin Mohammadiasl, the translator. If it had not been to her resistant and insistant efforts to contact different centers and send different emails to almost all dermatology centers in the world, I could not have been able to introduce this treatment to you, my colleagues and to vitiligo patients.

\section{REFERENCES}

[1] Autoimmne Melanocyte Destruction in Vitiligo (Published in August the first 2001)- Laboratory Investigation 81, 1061- 1068.

[2] Function Blocking Autoantibodies to the MelaninConcentrating Hormone Receptor in Vitiligo Patients (Published 08 May, 2006, Laboratoty Investigation , 86, 781-784 (2006)

[3] Magazine of Iranian Medical Science, no 43, Winter 2004.

[4] www.daroyab. ir/ G4538/ Pabak.

\section{The Note of the Translater}

Translating this article was an all compassing and time consuming projectand benefited from the input of many individuals. I would love to offer my sincere appreciation to all those who were of great help and motivation to me. First of all, I would like to thank my parents who taught me honesty, love of humanity and hope for a better life for every single individual. Secondly, I would love to thank my dear soulmate, Burak Ramazan Gungor, who brought happiness into my life. Finally,I would like to present my respect to one of the most hardworking women I have ever known in my life, who could achieve great goals in spite of all the limitations faced in the Middle East countries, a woman with great achievements... Gulsen Baykal.

\section{Translator}

Nooshin Mohammadiasl,

Urmia, Iran.

Citation: Akbar Mohammadrezaei, Nooshin Mohammadiasl. A Permanent Cure for Vitiligo: Immigration of Healthy Skin Cells to Unhealthy Sides of the Skin in Vitiligo through a New Treatment. Archives of Dermatology and Skin Care. 2018; 1(2): 09-14.

Copyright: (c) 2018 Akbar Mohammadrezaei, Nooshin Mohammadiasl. This is an open access article distributed under the Creative Commons Attribution License, which permits unrestricted use, distribution, and reproduction in any medium, provided the original work is properly cited. 